\title{
Generalized Exponential Euler Polynomials and Exponential Splines
}

\author{
Tian-xiao He \\ Department of Mathematics and Computer Science, Illinois Wesleyan University, Bloomington, USA \\ E-mail: the@iwu.edu \\ Received April 7, 2011, revised May 4, 2011, accepted May 20, 2011
}

\begin{abstract}
Here presented is constructive generalization of exponential Euler polynomial and exponential splines based on the interrelationship between the set of concepts of Eulerian polynomials, Eulerian numbers, and Eulerian fractions and the set of concepts related to spline functions. The applications of generalized exponential Euler polynomials in series transformations and expansions are also given.
\end{abstract}

Keywords: Eulerian Polynomial, Eulerian Number, Eulerian Fraction, Exponential Euler Polynomial, Euler-Frobenius Polynomial, B-Spline, Exponential Spline

\section{Introduction}

Eulerian polynomial is defined by the following equation (cf. for examples, [1-3]):

$$
\sum_{\ell \geq 0} \ell^{n} u^{\ell}=\frac{E_{n}(u)}{(1-u)^{n+1}}, \quad|u|<1 .
$$

It is well-known that the Eulerian polynomial, $E_{n}(u)$, of degree $n$ can be written in the form

$$
E_{n}(u)=\sum_{k=1}^{n} E(n, k) u^{k}, E_{0}(u)=1,
$$

where $E(n, k)$ are called the Eulerian numbers that can be calculated by using

$$
E(n, k)=\sum_{j=0}^{k}(-1)^{j}\left(\begin{array}{c}
n+1 \\
j
\end{array}\right)(k-j)^{n} \quad 1 \leq k \leq n ; E(n, 0)=1 .
$$

The Eulerian number $E(n, k)$ gives the number of permutations of $\{1,2, \cdots, n\}$ with $k-1$ permutation ascents ( $c f$. [4]), or equivalently, the number of permutation runs of length $k-1$ (cf. [3]), where a set of ascending sequences in a permutation is called a run and $j$ is a permutation ascent if the $j$ th term in the permutation less than the $j+1$ st term. A geometric interpretation of Eulerian number $E(n, k)$ were presented in [5] and [6], where which was found equaling to the volume of $k$ th slice of a $n$-dimensional unit cube, namely, $\left\{x \in[0,1]^{n}: k-1 \leq \sum_{j=1}^{n} x_{j} \leq k\right\}$. It is wellknown that the Eulerian fraction is a powerful tool in the study of the Eulerian numbers, Eulerian polynomial, Euler function and its generalization, Jordan function ( $c f$. [7]). The classical Eulerian fraction, $\alpha_{m}(x)$, can be expressed in the form

$$
\alpha_{n}(u):=\frac{E_{n}(u)}{(1-u)^{n+1}}, \quad u \neq 1 .
$$

$B$ spline functions are probably the most applicable one-dimensional polynomial spline functions. The values of $B$ spline functions can be defined as the volumes of slices of a $n$-dimensional cube (cf. [8]). Hence, one can expect a tight connection between the Eulerian numbers and the $B$ spline function values. This is is one of the motivations to study the interrelationship between Eulerian polynomials and B-splines. Another motivation is from the construction of the exponential splines by using the exponential Euler polynomial discussed in [9], which will be described in this paper.

Polynomial spline functions can be considered as broken polynomials with certain smoothness, which are used to overcome the stiffness of polynomials, for instance the Runge phenomenon. One way to construct the univariate polynomial B-splines is using divided differences to truncated powers, and the truncated powers can be generated by iterated integrations. An extension called the truncated Tchebycheff functions can be constructed similarly using the iterated integrations with respect to positive, smooth weight functions, and the Tchebycheffian B-splines are linear combination of these 
truncated Tchebycheff functions. Exponential splines belong to a class of Tchebycheffian B-splines, which preserve many features of the polynomial B-splines such as smoothness, compact support, non-negativity, and local linear independence. One may find more details in $[7,10,11]$ and $[12]$ and some applications in [13] and [14]. In [15], Dyn and Ron considered periodic exponential B-splines defined by weight functions $w_{i}(u)=\mathrm{e}^{a_{i} u} r_{i}(u)$ with $r_{i}(u+1)=r_{i}(u), a_{i} \in \mathbb{R}$, and showed these B-splines possess a significant property of translation invariant and satisfy a generalized Hermite-Genocchi formula. Motivated by this work, Ron define the higher dimensional $n$-directional exponential box splines in [16].

Another approach to construct the exponential splines to the base $u$ is using a linear combination of the translations of a polynomial B-spline with combination coefficients $u^{j}$. The exponential splines to the base $u$ can be presented and evaluated by using exponential Euler polynomials, which connect to some famous generating functions (GF's) in the combinatorics. The details can be found in Lecture 3 of [9-11], and Chapter 2 of [17], and we shall briefly quote them in next section. This approach can be used to derive a strong interrelationship between two different fields. For instance, in [18], the author presents the equivalence between exponential Euler polynomials, Euler-Frobenius polynomials, etc. and some frequently used concepts in combinatorics such as Eulerian fractions, Eulerian polynomials, etc., respectively, which will be surveyed as follows.

Let $n$ be an integer, $n \geq 0$. The symbol $S_{n}$ denotes the class of splines of order $n$, i.e., functions $s(x)$ satisfying the following conditions: (1) $s(x) \in C^{n-1}(\mathbb{R})$ and (2) $s(x) \in \pi_{n}$ in each interval $(j, j+1)$,

$j=0, \pm 1, \pm 2, \cdots$, where $\pi_{n}$ is the class of polynomials of degree not exceeding $n$.

In the central part of Schoenberg's lectures on "Cardinal Spline Interpolation" (CSI) (cf. [9]), the exponential Euler polynomial of degree $n$ to the base $u$ is defined as

$$
A_{n}(x ; u):=n !\left(1-u^{-1}\right)^{-n} \phi_{n}(x ; u), 0 \leq x \leq 1, u \neq 0,1,(1.5)
$$

where

$$
\phi_{n}(x ; u):=\sum_{-\infty}^{\infty} u^{j} Q_{n+1}(x-j)
$$

is the exponential spline of degree $n$ to the base $u$, where

$$
Q_{n}(x):=M(x ; 0,1, \cdots, n)
$$

denote the cardinal forward B-splines. Here, an exponential spline $f \in S_{n}$ means an element in $S_{n}$ satisfying the functional equation

$$
f(x+1)=f(x) .
$$

Obviously, $Q_{n}(x) \in S_{n-1}$. Therefore, $\phi_{n} \in S_{n}$, and it is easy to find $\phi_{n}$ satisfies (1.6).

From [9], $\phi_{n}(x ; u)$ satisfies $\phi_{n}(x+1 ; u)=u \phi(x ; u)$ and is a polynomial in the interval $0<x<1$ with the form

$$
\phi_{n}(x ; u)=\frac{1}{n !}\left(1-u^{-1}\right)^{n} x^{n}+\text { lower degree terms }
$$

for $0<x<1$. Hence, $A_{n}(x ; u)$ is a monic polynomial in $0 \leq x \leq 1$. From [9], the generating function of $\left\{A_{n}(x ; u)\right\}$ is

$$
\frac{u-1}{u-\mathrm{e}^{t}} \mathrm{e}^{x t}=\sum_{n \geq 0} A_{n}(x ; u) \frac{t^{n}}{n !} .
$$

Using (1.7) we may expand both sides of

$$
\frac{u-1}{u-\mathrm{e}^{t}} \mathrm{e}^{x t}=\frac{1}{t} \frac{\mathrm{d}}{\mathrm{d} x}\left(\frac{u-1}{u-\mathrm{e}^{t}} \mathrm{e}^{x t}\right),
$$

and comparing the coefficients of power of $t$ in the expansions on two sides, we obtain

$$
A_{n}^{\prime}(x ; u)=n A_{n-1}(x ; u) .
$$

If $x=0$, then (1.7) reduces to

$$
\frac{u-1}{u-\mathrm{e}^{t}}=\sum_{n \geq 0} a_{n}(u) \frac{t^{n}}{n !},
$$

where $a_{n}(u)=A_{n}(0 ; u)$. It is easy to see that

$$
A_{n}(x ; u)=\sum_{j=0}^{n}\left(\begin{array}{l}
n \\
j
\end{array}\right) a_{j}(u) x^{n-j} .
$$

In particular,

$$
A_{n}(1 ; u)=\sum_{j=0}^{n}\left(\begin{array}{l}
n \\
j
\end{array}\right) a_{j}(u) .
$$

Hence, we call $a_{n}(u)$ the coefficient (polynomials) of the exponential Euler polynomial $A_{n}(x ; u)$.

In addition, multiplying (1.9) by $u-\mathrm{e}^{z}$ and comparing the coefficients of the powers of $u$, we have the relations

$$
a_{0}(u)=1 u a_{n}(u)=\sum_{k=0}^{n}\left(\begin{array}{l}
n \\
k
\end{array}\right) a_{k}(u) .
$$

Jointing (1.11) and (1.12) yields

$$
A_{n}(1 ; u)=u A_{n}(0 ; u) \text {. }
$$

If $A_{n}^{(\ell)}(1 ; u)=u A_{n}^{(\ell)}(0 ; u)$ for all integer $n>0$, then (1.8) implies

$$
A_{n}^{(\ell+1)}(1 ; u)=n A_{n-1}^{(\ell)}(1 ; u)=n u A_{n-1}^{(\ell)}(0 ; u)=u A_{n}^{(\ell+1)}(0 ; u) .
$$

Thus, we have 


$$
A_{n}^{(\ell)}(1 ; u)=u A_{n}^{(\ell)}(0 ; u)
$$

for all integer $\ell>0$.

In the following, we call

$$
\Pi_{n}(u):=a_{n}(u)(u-1)^{n} \equiv A_{n}(0 ; u)(u-1)^{n}
$$

the Euler-Frobenius polynomial. Since

$$
\begin{aligned}
A_{n}(0 ; u) & :=n !\left(1-u^{-1}\right)^{-n} \phi_{n}(0 ; u) \\
& =n !\left(1-u^{-1}\right)^{-n} \sum_{-\infty}^{\infty} u^{j} Q_{n+1}(-j),
\end{aligned}
$$

$\Pi_{n}(u)$ defined by (1.15) can be written as

$$
\begin{aligned}
\Pi_{n}(u) & =n ! \sum_{-\infty}^{\infty} Q_{n+1}(-j) u^{n+j} \\
& =n ! \sum_{j=0}^{n-1} Q_{n+1}(n-j) u^{j} \\
& =n ! \sum_{j=0}^{n-1} Q_{n+1}(j+1) u^{j} .
\end{aligned}
$$

From (1.17), we know $\Pi_{n}(u)$ has $n-1$ zeros, all negative and simple, with $\lambda$ a zero if and only if $1 / \lambda$ is. A proof can be found, for example, in [17].

On the other hand, from (1.9) and (1.15) we have

$$
\frac{1}{e^{z}-u}=\sum_{n \geq 0} \frac{\Pi_{n}(u)}{(1-u)^{n+1}} \frac{(-z)^{n}}{n !} .
$$

Transferring $z$ to $-z$, we change the above equation as

$$
\frac{\mathrm{e}^{z}}{1-u \mathrm{e}^{z}}=\sum_{n \geq 0} \frac{\Pi_{n}(u)}{(1-u)^{n+1}} \frac{z^{n}}{n !} .
$$

The left-hand side of the above equation can be expanded as

$$
\frac{\mathrm{e}^{z}}{1-u \mathrm{e}^{z}}=\sum_{\ell \geq 0} u^{\ell} \mathrm{e}^{(\ell+1) z}=\sum_{\ell \geq 0} \sum_{n \geq 0}(\ell+1)^{n} u^{\ell} \frac{z^{n}}{n !}
$$

Comparing the right-hand sides of the last two equations yields

$$
\frac{\Pi_{n}(u)}{(1-u)^{n+1}}=\sum_{\ell \geq 0}(\ell+1)^{n} u^{\ell} .
$$

(1.18) implies

$$
\Pi_{n+1}(u)=(1+n u) \Pi_{n}(u)+u(1-u) \Pi_{n}^{\prime}(u) .
$$

In the introduction, we give the following definition of the Eulerian polynomial of degree $n$, which is similar to (1.18).

$$
\sum_{\ell \geq 0} \ell^{n} u^{\ell}=\frac{E_{n}(u)}{(1-u)^{n+1}},|u|<1,
$$

where the Eulerian polynomial, $E_{n}(u)$ can be written in the form of (1.2) with the Eulerian numbers calculated using (1.3).

In addition, Eulerian fraction, denoted by $\alpha_{n}(u)$, is defined by

$$
\alpha_{n}(u):=\frac{E_{n}(u)}{(1-u)^{n+1}}, u \neq 1
$$

Comparing (1.18) and (1.20), (1.17) and (1.7), and (1.15) and (1.3), [18] gives the following results.

Proposition 1.1 [18] Let $\Pi_{n}(u)$ and $a_{n}(u)$ be the polynomials defined by (1.15) and (1.9), respectively, and let Eulerian polynomials $E_{n}(u)$, Eulerian numbers $E(n, k)$, and Eulerian fractions $a_{n}(u)$ be defined by (1.20), (1.2), and (1.21), respectively. Denote the cardinal forward B-spline of order $n$ by $Q_{n}(u)$. Then we can set the relationship between the concepts as

$$
\begin{gathered}
E_{n}(u)= \begin{cases}\Pi_{n}(u)=1 & \text { if } n=0 \\
u \Pi_{n}(u) & \text { if } n>0,\end{cases} \\
E(n, k)=n ! Q_{n+1}(k)(n>0), \\
a_{n}(u)=\left\{\begin{array}{ll}
(1-u) \alpha_{n}(u)=1 & \text { if } n=0 \\
\frac{1-u}{u}(-1)^{n} \alpha_{n}(u) & \text { if } n>0
\end{array} .\right.
\end{gathered}
$$

As examples of the interrelationships shown in (1.22) (1.24), we see
1) $(n=0) \Pi_{0}(u)=1, E_{0}(u)=1$;
2) $(n=1) \Pi_{1}(u)=1, E_{1}(u)=u$;
3) $(n=2) \Pi_{2}(u)=1+u, E_{2}(u)=u+u^{2}$; etc.

and

1) $(n=1)\{E(1, k)\}=\{0,1,0\}, \quad\left\{Q_{2}(k)\right\}=\{0,1,0\}$;

2)

$(n=2)\{E(2, k)\}=\{0,1,1,0\},\left\{Q_{3}(k)\right\}=\left\{0, \frac{1}{2}, \frac{1}{2}, 0\right\}$

3)

$(n=3)\{E(3, k)\}=\{0,1,4,1,0\}, \quad\left\{Q_{4}(k)\right\}=\left\{0, \frac{1}{6}, \frac{2}{3}, \frac{1}{6}, 0\right\} ;$ etc.

Using Proposition 1.1, [18] derives many properties of B-spline and Euler-Frobenius polynomials readily from the corresponding properties of Eulerian polynomials and Eulerian numbers, and vice versa. Applications in B-spline interpolation and evaluation of Riemann Zeta function values at odd integers are also given in [18].

Let $u \neq 0,1$. It is well-known that the Eulerian fraction defined by (1.21) can be written as

$$
\alpha_{n}(u)=\sum_{j=0}^{n} j ! S(n, j) \frac{u^{j}}{(1-u)^{j+1}},
$$


where $S(n, j)$ are the Stirling numbers of the second kind, i.e., $j ! S(n, j)=\left[\Delta^{j} t^{n}\right]_{t=0}$, or equivalently, the number of partitions of $n$ distinct elements in $j$ blocks.

The connection shown in Proposition 1.1 also provides some important applications, shown in Section 2, of $\mathrm{B}$-splines and exponential B-splines to the enumerative combinatorics. In the section, a generalized Eulerian fraction and a generalized exponential spline to the base $u$ are derived. Section 3 discusses the applications of the generalized exponential Euler polynomials in the series transformations and expansions. Furthermore, using the combinatorial technique, we may generate exponential splines in higher dimensional setting, which will be briefly described at the end of the section and will be discussed in a later paper on the multivariate spline functions.

\section{Generalized Exponential Splines}

In this section, we will introduce a generalized Eulerian fraction, from which a generalized exponential spline to the base $u$ will be derived. We shall also discuss the corresponding generalized Euler-Frobenius polynomials and their applications.

It is obvious that (1.25) is equivalently

$$
\alpha_{n}(u)=\sum_{j=0}^{n} S(n, j)\left((1-u)^{-1}\right)^{(j)} u^{j},
$$

where $u \neq 0,1$. Hence, we may extend the Eulerian fraction to a more general case, which will be denoted by $\alpha_{n}(u \mid g(u))$ and called generalized Eulerian fractions associated with an infinitely differentiable function $g(u)$ :

$$
\alpha_{n}(u \mid g(u)):=\sum_{j=0}^{n} S(n, j) g^{(j)}(u) u^{j}
$$

Therefore, $\alpha_{n}(u) \equiv \alpha_{n}\left(u \mid(1-u)^{-1}\right)$. An analogue of the coefficients of the exponential Euler polynomials $a_{n}(u)$ defined by (1.10) is extended to

$$
a_{n}(u \mid g(u)):=\left\{\begin{array}{ll}
\frac{1}{g(u)} \alpha_{n}(u \mid g(u))=1 & \text { if } n=0 \\
\frac{1}{g(u)-1}(-1)^{n} \alpha_{n}(u \mid g(u)) & \text { if } n>0
\end{array},\right.
$$

which is associated with an analytic function $g(u)$.

We now find the generating function of $\left\{\alpha_{n}(u \mid g(u))\right\}$ and $a_{n}(u \mid g(u))$.

Theorem 2.1 Let $g(u)$ be an infinitely differentiable function, and let $\alpha_{n}(u \mid g(u))$ and $a_{n}(u \mid g(u))$ be defined by (2.2) and (2.3), respectively. Then their exponential generating functions are

$$
g\left(u \mathrm{e}^{t}\right)=\sum_{n \geq 0} \alpha_{n}(u \mid g(u)) \frac{t^{n}}{n !}
$$

and

$$
\frac{g\left(u \mathrm{e}^{-t}\right)-1}{g(u)-1}=\sum_{n \geq 0} a_{n}(u \mid g(u)) \frac{t^{n}}{n !},
$$

where $u \notin\{u: g(u)=1\}$.

Proof. Substituting (2.2) into the right-hand side of (2.4) and using the generalized Euler series transform formula (cf. [19] and (3.1) in [20]), we can evaluate the exponential generating function of $\alpha_{n}(u \mid g(u))$ formally as

$$
\begin{aligned}
& \sum_{n \geq 0} \alpha_{n}(u \mid g(u)) \frac{t^{n}}{n !}=\sum_{n \geq 0} \sum_{j=0}^{n} S(n, j) g^{(j)}(u) u^{j} \frac{t^{n}}{n !} \\
& =\sum_{n \geq 0} \sum_{j=0}^{n} \frac{\left.\Delta^{j} u^{n}\right|_{u=0}}{j !} g^{(j)}(u) u^{j} \frac{t^{n}}{n !}=\sum_{n \geq 0}\left(\sum_{j \geq 0} j^{n} g^{(j)}(u) \frac{u^{j}}{j !}\right) \frac{t^{n}}{n !} \\
& =\sum_{j \geq 0}\left(\sum_{n \geq 0} j^{n} \frac{t^{n}}{n !}\right) g^{(j)}(u) \frac{u^{j}}{j !}=\sum_{j \geq 0} e^{j t} g^{(j)}(0) \frac{u^{j}}{j !}=g\left(u e^{t}\right) .
\end{aligned}
$$

Similarly, by substituting (2.3) into the right-hand side of (2.5) and applying formula (2.4) to the resulting expression, we have

$$
\begin{aligned}
& \sum_{n \geq 0} a_{n}(u \mid g(u)) \frac{t^{n}}{n !} \\
& =1+\frac{1}{g(u)-1} \sum_{n \geq 1} \sum_{j=0}^{n}(-1)^{n} \alpha_{n}(u \mid g(u)) \frac{t^{n}}{n !} \\
& =1+\frac{1}{g(u)-1} \sum_{n \geq 0} \sum_{j=0}^{n}(-1)^{n} \alpha_{n}(u \mid g(u)) \frac{t^{n}}{n !}-\frac{g(u)}{g(u)-1} \\
& =\frac{1}{1-g(u)}+\frac{g\left(u \mathrm{e}^{-t}\right)}{g(u)-1},
\end{aligned}
$$

which yields (2.5) and completes the proof of the theorem.

From $a_{n}(u \mid g(u))$ we now construct polynomials $A(x ; u \mid g(u))$ for $0 \leq x \leq 1$ using the following expansion.

$$
\frac{g\left(u \mathrm{e}^{-t}\right)-1}{g(u)-1} \mathrm{e}^{x t}=\sum_{n \geq 0} A_{n}(x ; u \mid g(u)) \frac{t^{n}}{n !} .
$$

Thus, $A_{n}(x ; u \mid g(u))$ can be evaluated from $a_{n}(u \mid g(u))$ as follows.

$$
A_{n}(x ; u \mid g(u))=\sum_{j=0}^{n}\left(\begin{array}{c}
n \\
j
\end{array}\right) a_{j}(u \mid g(u)) x^{n-j}
$$


for $0 \leq x \leq 1$. In particular, $A_{n}\left(0 ; u \mid g(u)=a_{n}(u \mid g(u))\right.$ and

$$
A_{n}(1 ; u \mid g(u))=\sum_{j=0}^{n}\left(\begin{array}{c}
n \\
j
\end{array}\right) a_{j}(u \mid g(u))
$$

We call $A_{n}(x ; u \mid g(u))$ the generalized exponential Euler polynomial of degree $n$ associated with $g$ to the base $u$, and $a_{j}(u \mid g(u))$ the coefficient of $A_{n}(x ; u \mid g(u))$. In addition, we call $A_{n}(x ;-1 \mid g(-1))$ the generalized Euler polynomials, and $A_{n}(x ;-1)$, the regular Euler polynomials, are the special case of $A_{n}(x ;-1 \mid g(-1))$ for $g(u)=1 /(1-u)$.

A generalized exponential spline function to the base $u$, denoted by $\phi_{n}(x ; u \mid g(u))$, is therefore defined using $A_{n}(x ; u \mid g(u))(0 \leq x \leq 1)$ as follows.

$$
\phi_{n}(x ; u \mid g(u))=\frac{1}{n !}(1-g(u))^{-n} A_{n}(x ; u \mid g(u))
$$

in $0 \leq x \leq 1$. However, if one wants to extend $\phi_{n}(x ; u \mid g(u))$ to all real $x$ associated with $g(u)$ such that $\phi_{n} \in C^{n-1}, A_{n}(x ; u \mid g(u))$ needs to satisfy some periodic conditions. Let us consider the class of $g(u)=(1-a u)^{-\alpha-1}$ with real constants $\alpha$ and $a \neq 0$. Then we have the following result.

Theorem 2.2 Let function $\phi_{n}(x ; u \mid g(u))$ be defined by (2.8) on $0 \leq x \leq 1$ associated with $g(u)=(1-a u)^{-\alpha-1}$, where $a$ and $\alpha$ are real constants and $a \neq 0, \phi_{n}$ can be extended to a $C^{n-1}(\mathbb{R})$ continuous function for all real $x$ if and only if $\alpha=0$. Furthermore, the extension is fulfilled by the functional equation

$$
\phi_{n+1}\left(x+1 ; u \mid(1-a u)^{-1}\right)=\operatorname{au} \phi_{n}\left(x ; u \mid(1-a u)^{-1}\right) .
$$

Proof. From [9], for (2.9) combined with the condition that $\phi_{n}\left(x ; u \mid(1-a u)^{-\alpha-1}\right) \in C^{n-1}(\mathbb{R})$ require $A_{n}\left(x ; u \mid(1-a u)^{-\alpha-1}\right)$ to satisfy in $[0,1]$ the periodic conditions

$$
A_{n}^{(v)}\left(1 ; u \mid(1-a u)^{-\alpha-1}\right)=a u A_{n}^{(v)}\left(0 ; u \mid(1-a u)^{-\alpha-1}\right)
$$

for $v=0,1, \cdots, n-1$. From (2.7), we have

$$
\begin{aligned}
A_{n}^{(v)}(x ; u \mid g(u)) & =\frac{n !}{(n-v) !} \sum_{j=0}^{n-v}\left(\begin{array}{c}
n-v \\
j
\end{array}\right) a_{j}(u \mid g(u)) x^{n-v-j} \\
& =\frac{n !}{(n-v) !} A_{n-v}(x ; u \mid g(u)) .
\end{aligned}
$$

Hence, condition (2.10) is equivalent to

$$
\begin{aligned}
A_{n}\left(1 ; u \mid(1-a u)^{-\alpha-1}\right) & =a u A_{n}\left(0 ; u \mid(1-a u)^{-\alpha-1}\right) \\
& =a u a_{n}\left(u \mid(1-a u)^{-\alpha-1}\right)
\end{aligned}
$$

for all $n \geq 1$. From (2.7) (2.3), and (2.2), we have

$$
\begin{aligned}
& A_{n}\left(1 ; u \mid(1-a u)^{-\alpha-1}\right) \\
= & 1+\frac{1}{(1-q u)^{-\alpha-1}-1} \sum_{j=1}^{n} \sum_{k=0}^{j}\left(\begin{array}{c}
n \\
j
\end{array}\right)(-1)^{j} S(j, k) \frac{k !(a u)^{k}}{(1-a u)^{\alpha+k+1}} \\
= & 1+\frac{1}{(1-q u)^{-\alpha-1}-1} \sum_{k=1}^{n} \sum_{j=k}^{n}\left(\begin{array}{c}
n \\
j
\end{array}\right)(-1)^{j} S(j, k) \frac{k !(a u)^{k}}{(1-a u)^{\alpha+k+1}} \\
= & 1+\frac{1}{(1-q u)^{-\alpha-1}-1}\left[\sum_{k=1}^{n}(-1)^{n} S(n, k) \frac{k !(a u)^{k}}{(1-a u)^{\alpha+k+1}}\right. \\
& \left.-\sum_{k=1}^{n} \sum_{j=k}^{n}\left(\begin{array}{c}
n \\
j
\end{array}\right)(-1)^{j}(k+1) S(j, k+1) \frac{k !(a u)^{k}}{(1-a u)^{\alpha+k+1}}\right],
\end{aligned}
$$

where the last step is due to the identities

$$
S(j+1, k+1)=S(j, k)+(k+1) S(j, k+1)
$$

and

$$
\sum_{j=k}^{n}\left(\begin{array}{l}
n \\
j
\end{array}\right)(-1)^{j} S(j+1, k+1)=(-1)^{n} S(n, k) .
$$

The double sum in the rightmost expression of (2.12) can be further changed to

$$
\begin{aligned}
& \sum_{k=1}^{n} \sum_{j=k}^{n}\left(\begin{array}{c}
n \\
j
\end{array}\right)(-1)^{j}(k+1) S(j, k+1) \frac{k !(a u)^{k}}{(1-a u)^{\alpha+k+1}} \\
= & \sum_{k=2}^{n} \sum_{j=k-1}^{n}\left(\begin{array}{c}
n \\
j
\end{array}\right)(-1)^{j} S(j, k) \frac{k !(a u)^{k-1}}{(1-a u)^{\alpha+k}} \\
= & \frac{1-a u}{a u}\left(\sum_{k=1}^{n} \sum_{j=k}^{n}\left(\begin{array}{c}
n \\
j
\end{array}\right)(-1)^{j} S(j, k) \frac{k !(a u)^{k}}{(1-a u)^{\alpha+k+1}}\right. \\
= & \left.\frac{1-a u}{a u} \sum_{j=1}^{n}\left(\begin{array}{c}
n \\
j
\end{array}\right)(-1)^{j}\left(\begin{array}{c}
n \\
j
\end{array}\right)(-1)^{j} S(j, k) \frac{a u}{(1-a u)^{\alpha+2}}\right) \\
& +\frac{1}{(1-a u)^{\alpha+1}},
\end{aligned}
$$

where the last step comes from $S(j, 1)=1$ for all $j \geq 1$. Substituting the last expression of the double sum

$$
\sum_{k=1}^{n} \sum_{j=k}^{n}\left(\begin{array}{l}
n \\
j
\end{array}\right)(-1)^{j}(k+1) S(j, k+1) \frac{k !(a u)^{k}}{(1-a u)^{\alpha+k+1}} \text { into }
$$


equation

$$
\begin{aligned}
& \sum_{k=1}^{n} \sum_{j=k}^{n}\left(\begin{array}{c}
n \\
j
\end{array}\right)(-1)^{j} S(j, k) \frac{k !(a u)^{k}}{(1-a u)^{\alpha+k+1}} \\
= & \sum_{k=1}^{n}(-1)^{n} S(n, k) \frac{k !(a u)^{k}}{(1-a u)^{\alpha+k+1}} \\
& -\sum_{k=1}^{n} \sum_{j=k}^{n}\left(\begin{array}{c}
n \\
j
\end{array}\right)(-1)^{j}(k+1) S(j, k+1) \frac{k !(a u)^{k}}{(1-a u)^{\alpha+k+1}},
\end{aligned}
$$

which comes from (2.12), and solving the resulting equation for $\sum_{k=1}^{n} \sum_{j=k}^{n}\left(\begin{array}{l}n \\ j\end{array}\right)(-1)^{j} S(j, k) \frac{k !(a u)^{k}}{(1-a u)^{\alpha+k+1}}$, we obtain

$$
\begin{aligned}
& \sum_{k=1}^{n} \sum_{j=k}^{n}\left(\begin{array}{l}
n \\
j
\end{array}\right)(-1)^{j} S(j, k) \frac{k !(a u)^{k}}{(1-a u)^{\alpha+k+1}} \\
& =a u \sum_{k=1}^{n}(-1)^{n} S(n, k) \frac{k !(a u)^{k}}{(1-a u)^{\alpha+k+1}}-\frac{a u}{(1-a u)^{\alpha+1}} .
\end{aligned}
$$

Substituting the last equation to the first equation of (2.12) yields

$$
\begin{aligned}
& A_{n}\left(1 ; u \mid(1-a u)^{-\alpha-1}\right) \\
&=1+\frac{a u}{(1-a u)^{-\alpha-1}-1} \sum_{k=1}^{n}(-1)^{n} S(n, k) \frac{k !(a u)^{k}}{(1-a u)^{\alpha+k+1}} \\
&-\frac{a u}{1-(1-a u)^{\alpha+1}} \\
&= 1-\frac{a u}{1-(1-a u)^{\alpha+1}}+a u A_{n}\left(0 ; u \mid(1-a u)^{-\alpha-1}\right),
\end{aligned}
$$

where the last step is implied by

$$
\begin{aligned}
& A_{n}\left(0 ; u \mid(1-a u)^{-\alpha-1}\right)=a_{n}\left(u \mid(1-a u)^{-\alpha-1}\right) \\
& =\frac{(-1)^{n}}{(1-a u)^{-\alpha-1}-1} \sum_{k=1}^{n} S(n, k) \frac{k !(a u)^{k}}{(1-a u)^{\alpha+k+1}}
\end{aligned}
$$

(see (2.2) and (2.3). Therefore, if $A_{n}\left(1 ; u \mid(1-a u)^{-\alpha-1}\right)=a u A_{n}\left(0 ; u \mid(1-a u)^{-\alpha-1}\right)$, then $\alpha=0$. The sufficiency is obvious. This completes the proof of the theorem.

Remark 3.1 Other generalized exponential splines are planed to be constructed. And the properties of the exponential splines need to be investigated.

\section{Applications in Series Transformations and Expansions}

All formulas presented in this section are formal iden- tities in which we always assume that $x \neq 1$ or $x \neq-1$ according as $(1-x)^{-1}$ or $(1+x)^{-1}$ appears in the formulas.

Theorem 3.1 Let $g(u)$ and $h(u)$ be infinitely differentiable on $[0, \infty)$, and let $A_{k}(x ; u \mid g(u))(0 \leq x \leq 1)$ be the generalized exponential Euler polynomial to the base $u$ defined by (2.6) or (2.7). Then we have formally

$$
\begin{aligned}
& \sum_{k=1}^{\infty}(h(k)-h(0)) g^{(k)}(0) \frac{u^{k}}{k !} \\
& =(g(u)-1) \sum_{k=1}^{\infty} \frac{(-1)^{k}}{k !} h^{(k)}(0) A_{k}(0 ; u \mid g(u)),
\end{aligned}
$$

where $u$ is not any poles of $g(u)$ and $A_{k}(0 ; u \mid g(u))$ for any $k \geq 1$.

Proof. First, we apply the operator $g(u E)$ to the infinitely differentiable function $h(t)$ at $t=0$, where $E$ is the shift operator.

$$
\begin{aligned}
\left.g(u E) h(t)\right|_{t=0} & =\left.\sum_{k=0}^{\infty} \frac{1}{k !} g^{(k)}(0)(u E)^{k} h(t)\right|_{t=0} \\
& =\sum_{k=0}^{\infty} h(k) g^{(k)}(0) \frac{u^{k}}{k !} .
\end{aligned}
$$

On the other hand, we can present

$$
\begin{aligned}
\left.g(u E) h(t)\right|_{t=0} & =\left.g\left(u e^{D}\right) h(t)\right|_{t=0} \\
& =\left.\sum_{j=0}^{\infty} \frac{1}{j !} g^{(j)}(0)\left(u e^{D}\right)^{j} h(t)\right|_{t=0} \\
& =\sum_{j=0}^{\infty} \frac{u^{j}}{j !} g^{(j)}(0) \sum_{k=0}^{\infty} \frac{j^{k}}{k !} h^{(k)}(0) \\
& =\sum_{k=0}^{\infty}\left(\sum_{j=0}^{\infty} g^{(j)}(0) j^{k} \frac{x^{j}}{j !}\right) \frac{1}{k !} h^{(k)}(0) .
\end{aligned}
$$

By applying (3.1) in [19] to the inner sum of the rightmost side of the above equation for $f(t)=t^{k}$ and noting $S(k, j)=\left(\Delta^{j} t^{k}\right)_{t=0} / j$ !, we obtain

$$
\begin{aligned}
& \left.g(u E) h(t)\right|_{t=0} \\
& =\sum_{k=0}^{\infty}\left(\sum_{j=0}^{k}\left(\Delta^{j} t^{k}\right)_{t=0} g^{(j)}(u) \frac{u^{j}}{j !}\right) \frac{1}{k !} h^{(k)}(0) \\
& =\sum_{k=0}^{\infty}\left(\sum_{j=0}^{k} S(k, j) g^{(j)}(u) u^{j}\right) \frac{1}{k !} h^{(k)}(0) \\
& ={ }^{(*)} \sum_{k=0}^{\infty} \alpha_{k}(u \mid g(u)) \frac{1}{k !} h^{(k)}(0) \\
& ={ }^{(* *)} h(0) g(u)+(g(u)-1) \sum_{k=1}^{\infty} \frac{(-1)^{k}}{k !} h^{(k)}(0) a_{k}(u \mid g(u)),
\end{aligned}
$$

which implies (3.1) by writing

$h(0) g(u)=\sum_{k \geq 0} h(0) g^{(k)}(0) u^{k} / k$ ! formally, where for $(*)$ and $(* *)$ we use (2.2) and (2.3), respectively. 
This completes the proof of the theorem.

Remark 4.1 The series transformation formulas (3.1) have numerous applications by setting different infinitely differentiable functions for $g(x)$. For instance, If $g(u)=(1-u)^{-1}$, then (3.1) becomes to

$$
\sum_{k=1}^{\infty}(h(k)-h(0)) u^{k}=\sum_{k=1}^{\infty} h^{(k)}(0)\left(\frac{u}{1-u}\right)^{k+1} \phi_{k}(0 ; u),
$$

where $u \neq 1$ and the relation (1.5) has been used.

Evidently, when $h(u)$ is a polynomial, formulas (3.1) and (3.2) become closed form of summation formulas with a finite number of terms. Moreover, the Right-hand side of each formula may also be viewed as a $G F$ for the sequence of coefficeients contained in the power series on the left-hand side. For example, if $h(u)=u$, noting

$$
\phi_{k}(0 ; u)=\sum_{j=1}^{k} u^{-j} Q_{k+1}(j),
$$

we have

$$
\sum_{k=1}^{\infty} k u^{k}=\frac{u}{(1-u)^{2}} .
$$

At the end of this section, we will show a way to extend our construction of exponential splines to the higher dimensional setting. First, we shall adopt the multi-index notational system. Denote

$$
\begin{gathered}
\hat{t} \equiv\left(t_{1}, \cdots, t_{r}\right), \hat{x} \equiv\left(x_{1}, \cdots, x_{r}\right), \\
|\hat{t}|=\left(t_{1}+\cdots+t_{r}\right) \hat{t}+\hat{x} \equiv\left(t_{1}+x_{1}, \cdots, t_{r}+x_{r}\right), \\
\hat{u} \equiv\left(u_{1}, \cdots, u_{r}\right), \hat{0} \equiv(0, \cdots, 0), \\
\widehat{g(u)}=\prod_{i=1}^{r} g_{i}\left(u_{i}\right), \\
\frac{g\left(u e^{-t}\right)}{g(u)-1}=\prod_{i=1}^{r} \frac{g_{i}\left(u_{i} \mathrm{e}^{-|\hat{t}|}\right)-1}{g_{i}\left(u_{i}\right)-1}, \\
\hat{x} \cdot \hat{t} \equiv \sum_{i=1}^{r} x_{i} t_{i},
\end{gathered}
$$

Moreover, we write $t^{\lambda} \equiv t_{1}^{\lambda_{1}} \cdots t_{r}^{\lambda_{r}}$ with $\lambda \equiv\left(\lambda_{1}, \cdots, \lambda_{r}\right), r$ being non-negative integers. Also, $\lambda \geq 0$ means $\lambda_{i} \geq 0 \quad(i=1, \cdots, r)$, and $\lambda \geq \mu$ means $\lambda_{i} \geq \mu_{i}$ for all $i=1, \cdots, r$.

We now define a generalized higher dimensional exponential polynomials $A_{\lambda}(\widehat{x ; u \mid g(u)})$ as follows.

Definition 3.2 Let $g_{i}\left(u_{i}\right)$ be any given formal power series over the complex number field $\mathbb{C}$ with $g_{i}(0)=1$.
Then $A_{\lambda}(\widehat{x ; u \mid g(u)})$ defined by

$$
\begin{aligned}
\frac{\widehat{g\left(u \mathrm{e}^{-t}\right)}-1}{g(u)-1} \mathrm{e}^{\hat{x}, \hat{t}} & \equiv \prod_{i=1}^{r} \frac{g_{i}\left(u_{i} \mathrm{e}^{-|\hat{t}|}\right)-1}{g_{i}\left(u_{i}\right)-1} \mathrm{e}^{\hat{x}, \hat{t}} \\
& =\sum_{\lambda \geq 0} A_{\lambda}(\hat{x}, \hat{u}, \widehat{g(u)}) \frac{t^{\lambda}}{\lambda !}
\end{aligned}
$$

are called the generalized higher dimensional exponential Euler polynomials of degree $\lambda$ associated with $g$ to the base $\hat{u}$, where $\hat{u} \in[0,1]^{r}$. In particular, for $g(u)=1 /(1-u),(3.3)$ defines higher dimensional exponential Euler polynomials $A_{\lambda}(\hat{x} ; \hat{u})$ of degree $\lambda$ to the base $\hat{u}$ by

$$
\Pi_{i=1}^{r} \frac{u_{i}-1}{u_{i}-\mathrm{e}^{|\hat{t}|}} \mathrm{e}^{\hat{\hat{x}} \hat{t}}=\sum_{\lambda \geq 0} A_{\lambda}(\hat{x} ; \hat{u}) \frac{t^{\lambda}}{\lambda !} .
$$

Let $\hat{u}=(-1,-1, \ldots,-1)$. Then the polynomial $A_{\lambda}(\hat{x} ;(-1, \cdots,-1))$ defined by (3.4) is called the higher dimensional (regular) Euler polynomial of degree $\lambda$. The more details will be given in a later paper.

\section{Acknowledgments}

The author greatly appreciate the discussion with Carl deBoor since 2005 and the valuable suggestions and the lecture [17] he offered fervently.

\section{References}

[1] L. Carlitz, D. P. Roselle and R. Scoville, "Permutations and Sequences with Repetitions by Number of Increase," Journal of Combinatorial Theory, Vol. 1, No. 3, 1966, pp. 350-374. doi:10.1016/S0021-9800(66)80057-1

[2] L. Carlitz, "Eulerian Numbers and Polynomials of Higher Order," Duke Mathematical Journal, Vol. 27, 1960, PP. 401-423.

[3] L. Comtet, "Advanced Combinatorics: The Art of Finite and Infinite expansions," Springer, Dordrecht, 1974.

[4] R. L. Graham, D. E. Knuth and O. Patashnik, "Concrete Mathematics," A Foundation for Computer Science, 2nd Ed, Addison-Wesley, 1994, pp. 267-272.

[5] D. Foata, "Distribution Eulérienne et Mahoniennes sur le groups des permutations," Proceedings of the NATO Advanced Study Institute, Berlin, September 1-10, 1976, pp 27-49.

[6] R. P. Stanley, "Eulerian Partitions of a Unit Hypercube," Proceedings of the NATO Advanced Study Institute, Boston, 1977, p. 49.

[7] G. Birkhoff and C. de Boor, "Piecewise Polynomial Interpolation and Approximation," Approximation of Functions, Elsevier Publishing Company, Amsterdam, 1964, pp. 164-190.

[8] C. de Boor, K. Hllig, S. Riemenschneider, "Box Splines. 
Applied Mathematical Sciences," Springer-Verlag, New York, 1993.

[9] I. J. Schoenberg, "Cardinal Spline Interpolation," CBMS-NSF Regional Conference Series in Applied Mathematics, 1973.

[10] C. de Boor, "On the Cardinal Spline Interpolant to $e^{\text {iut }}$," SIAM Journal on Mathematics Analysis, Vol. 7, No. 6, 1976, pp. 930-941. doi:10.1137/0507073

[11] C. de Boor, "A Practical Guide to Splines," Revised edition. Applied Mathematical Sciences, Springer-Verlag, New York, 2001.

[12] L. Schumaker, "Spline Functions: Basic Theory," John Wiley \& Sons, New York, 1981.

[13] O. A. Vasicek and H. G. Fong, "Term Structure Modeling Using Exponential Splines," The Journal of Finance, Vol. 37, No. 2, pp. 339-348. doi:10.2307/2327333

[14] C. Zoppou, S. Roberts and R. J. Renka, "Exponential Spline Interpolation in Characteristic Based Scheme for Solving the Advective-Diffusion Equation," International Journal of Numerical Methods in Fluids, Vol. 33, No. 3, 2000 , pp. 429-452. doi:10.1002/1097-0363(20000615)33:3<429::AID-FLD6
0>3.0.CO;2-1

[15] N. Dyn, and A. Ron, "Recurrence Relations for Tchebycheffian B-splines," Journal d'Analyse Mathématique, Vol. 51, 1988, pp.118-138. doi:10.1007/BF02791121

[16] A. Ron, "Exponential Box Splines," Constructive Approximation, Vol. 4, No. 4, 1988, pp. 357-378. doi:10.1007/BF02075467

[17] C. de Boor, "Chapter II. Splines with Uniform Knots," draft jul74, TeXed, 1997.

[18] T. X. He, "Eulerian polynomials and B-splines," ICCAM-2010, University of Leuven, Belgium, July 5-9, 2010.

[19] T. X. He, L. C. Hsu, P. J.-S. Shiue and D. C. Torney, “A Symbolic Operator Approach to Several Summation Formulas for Power Series," Journal of Computational and Applied Mathematics, Vol. 177, 2005, pp. 17-33. doi:10.1016/j.cam.2004.08.002

[20] T. X. He, L. C. Hsu, and P. J.-S. Shiue, "A Symbolic Operator Approach to Several Summation Formulas for Power Series II," Discrete Mathematics, Vol. 308, No.16, 2008, pp. 3427-3440. doi:10.1016/j.disc.2007.07.001 